\title{
Structure Determination of Triplet Diphenylcarbenes by In Situ X-Ray Crystallographic Analysis
}

\author{
Masaki Kawano, ${ }^{*}$, Katsuyuki Hirai, ${ }^{*}$ Hideo Tomioka, ${ }^{*} \xi$ and Yuji Ohashi ${ }^{*}$ \\ Department of Chemistry and Materials Science, Tokyo Institute of Technology, Tokyo 152-8551 Japan \\ $†$ CREST, Japan Science and Technology Corporation, Kawaguchi, Saitama 332-0012 Japan \\ $\ddagger$ Chemistry Department for Materials, Faculty of Engineering, and \\ Instrumental Analysis Facilities, Life Science Research Center, \\ Mie University, Tsu, Mie 514-8507 Japan \\ Present address: \\ "Departure of Applied Chemistry, School of Engineering, The University of Tokyo, 7-3-1 Hongo \\ Bunkyo-ku, Tokyo 113-8656, Japan \\ §Department of Applied Chemistry, Aichi Institute of Technology, \\ Toyota, Aichi 470-0392, Japan
}

\section{X-Ray Crystal Structures of Precursor Diazo Compounds.}

Crystals suitable for X-ray analysis were obtained for all the seven diphenyldiazomethanes (DDMs) in scheme 1. All cif files are deposited in CSD (CCDC $619889-619896)$. The ORTEP views of compounds 2- $\mathrm{N}_{2}-7-\mathrm{N}_{2}$ are shown in Figures $\mathrm{S} 1-6$, respectively, and crystallographic data are presented in Table S1 (a crystal structure of $\mathbf{1}-\mathrm{N}_{2}$ was reported in ref 20). Two independent molecules are observed in the unit cell for 5- $\mathrm{N}_{2}$ and $7-\mathrm{N}_{2}$, while one independent molecule exists in the unit cell for the other DDMs. The selected bond angles and bond lengths concerning the main framework of DDMs are listed in Table 2, where $\theta$ is the diazo carbon C-C-C angle $\left(^{\circ}\right)$ and the $\omega$ interplanar angle $\left(^{\circ}\right)$ between two phenyl rings, while $d_{1}$ and $d_{2}$ are the C-C distances $(\AA)$ between aromatic and diazo carbons and $d_{3}$ and $d_{4}$ are the distances $(\AA)$ between C- $\mathrm{N}_{1}$ and $\mathrm{N}_{1}-\mathrm{N}_{2}$, respectively (see Scheme 2). 
The most prominent structural difference between DDMs having no ortho substituents, i.e., 6- $\mathrm{N}_{2}$, and those bearing ortho substituents, i.e., $1-\mathrm{N}_{2}-5-\mathrm{N}_{2}$, in the main framework is seen in the interplanar angle $(\omega)$ between two phenyl rings. Thus, in $6-\mathrm{N}_{2}$, this angle is $46.61(3)^{\circ}$, while the angles in $2-\mathrm{N}_{2}$ to $5-\mathrm{N}_{2}$ are in the range of $82.46(5)$ to $87.74(2)^{\circ}$. As the angles of diazo carbon $(\theta)$ change very little on going from 6- $\mathrm{N}_{2}$ to $1-\mathrm{N}_{2}-5-\mathrm{N}_{2}$, the observation suggests that the steric repulsion between the ortho substituents is mitigated by rotating the phenyl ring along $\mathrm{C}\left(=\mathrm{N}_{2}\right)-\mathrm{C}(\mathrm{Ar})$ bond rather than by expanding the diazo carbon C-C-C angle. ${ }^{24}$ In this way, procarbenic diazo carbon is shielded more effectively from external reagents by protector. Diazofluorene $7-\mathrm{N}_{2}$ has a structure mostly dictated by unique structural requirements incorporated into a five-membered ring.

(A)

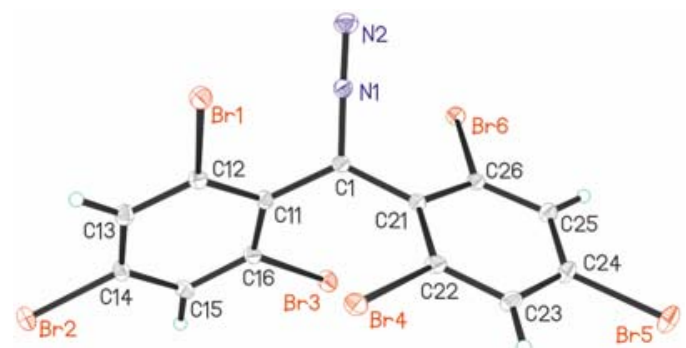

(B)

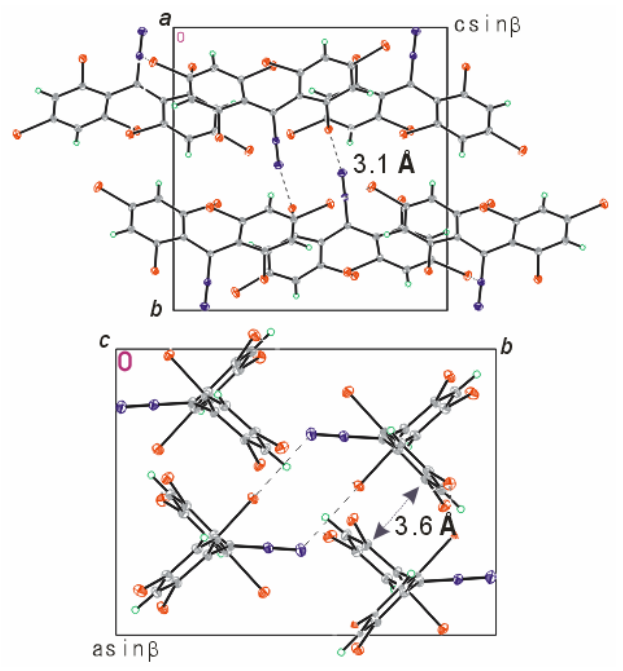

Figure S1. (A) Thermal ellipsoid (probability level 50\%) plot and (B) a packing diagram of bis(2,4,6tribromophenyl)diazomethane $\left(2-\mathrm{N}_{2}\right)$. 
(A)

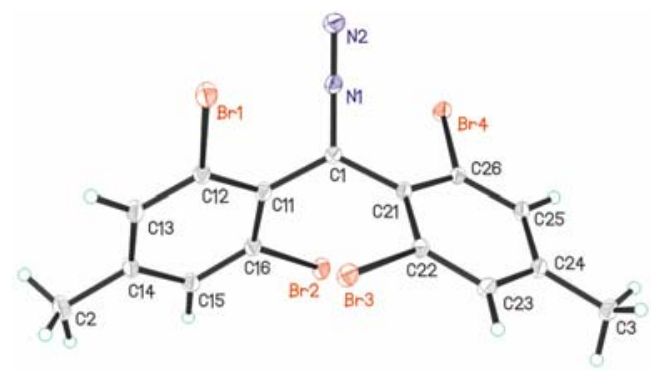

(B)

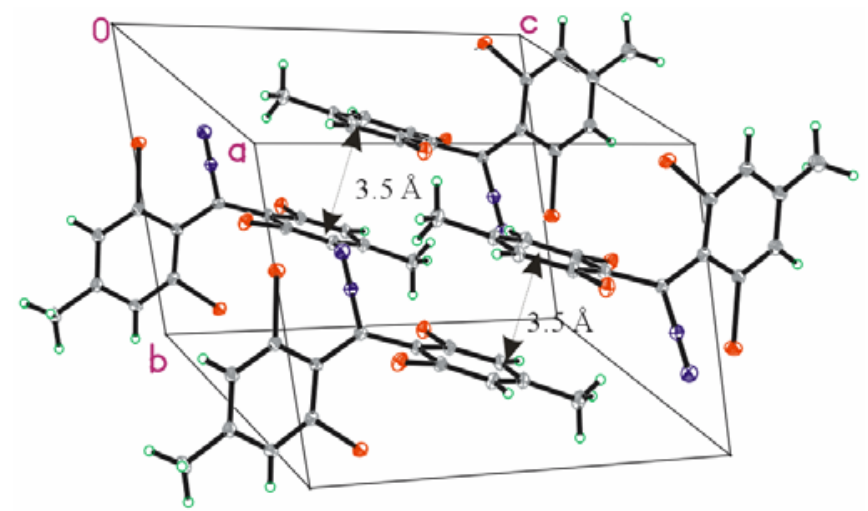

Figure S2. (A) Thermal ellipsoid (probability level 50\%) plot and (B) a packing diagram of bis(2,6dibromo-4-methylphenyl)diazomethane (3- $\left.\mathrm{N}_{2}\right)$. 
(A)

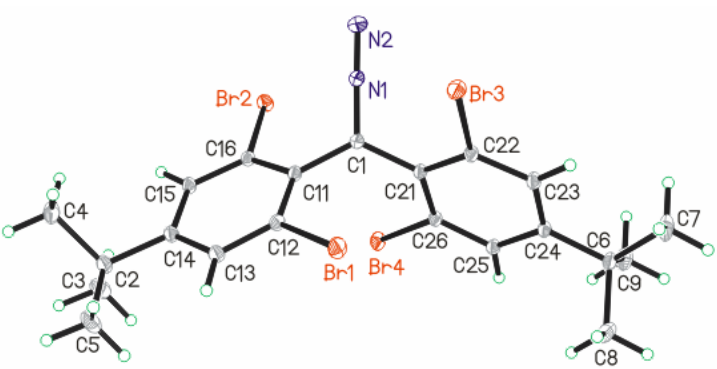

(B)

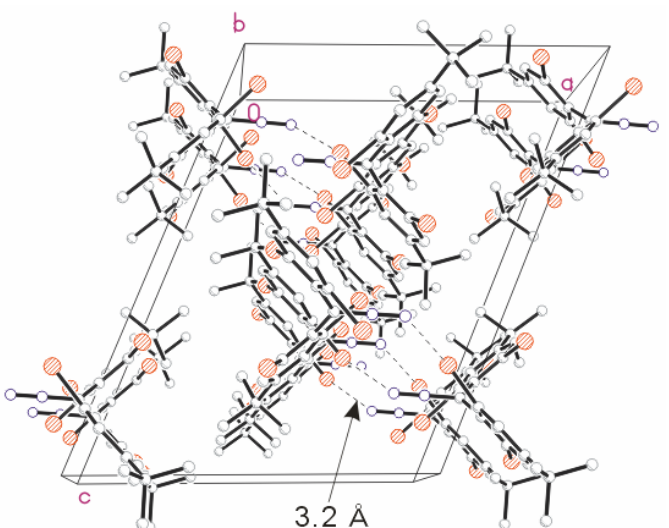

Figure S3. (A) Thermal ellipsoid (probability level 50\%) plot and (B) a packing diagram of bis(2,6dibromo-4-tert-butylphenyl)diazomethane (4- $\left.\mathrm{N}_{2}\right)$. 
(A)

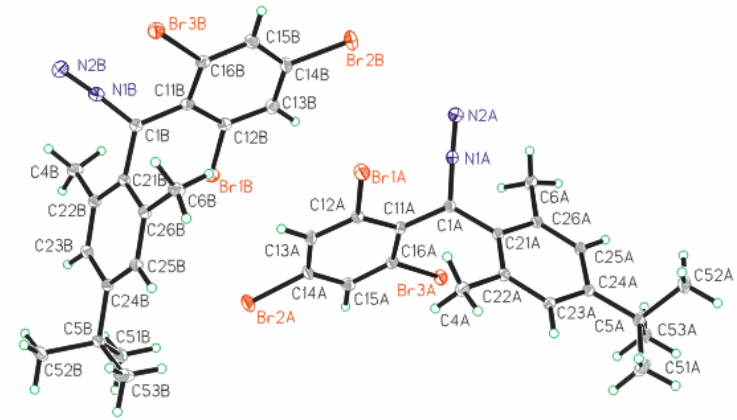

(B)

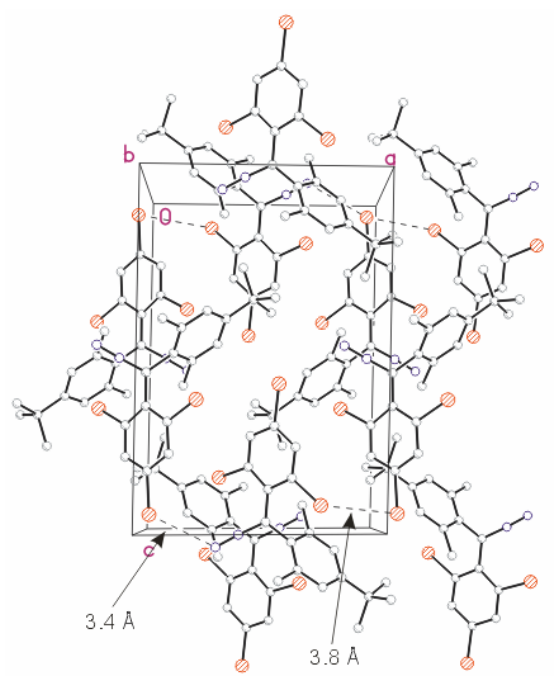

Figure S4. (A) Thermal ellipsoid (probability level 50\%) plot and (B) a packing diagram of 2,4,6triboromophenyl)(2,6-dimethyl-4-tert-butylphenyl)diazomethane (5- $\left.\mathrm{N}_{2}\right)$. 
(A)

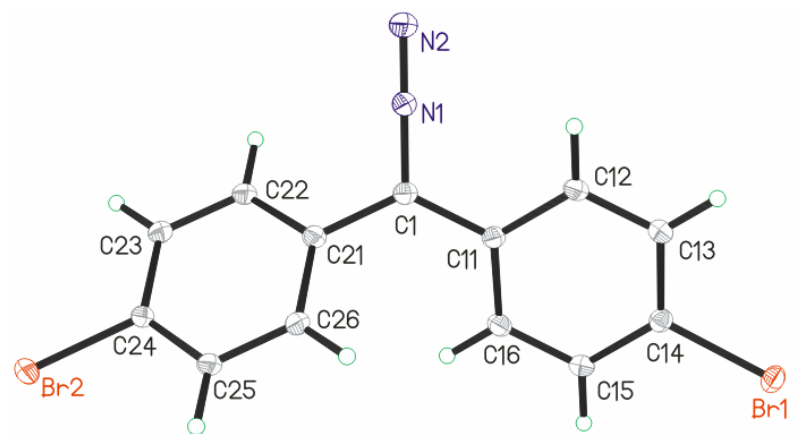

(B)

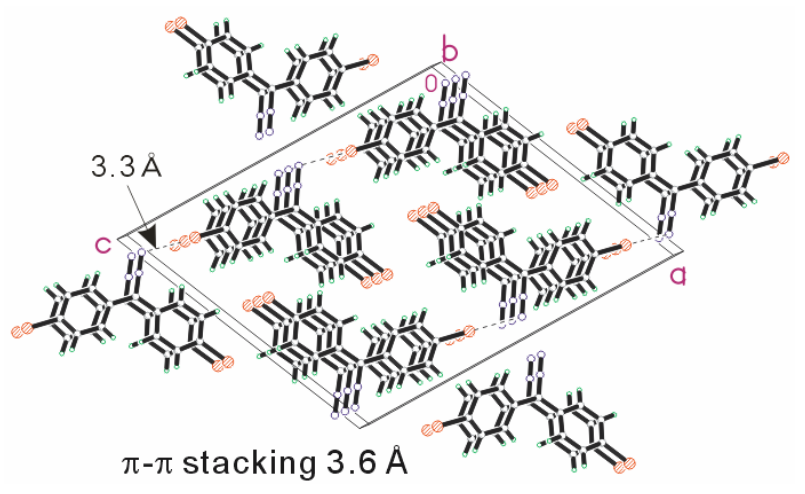

Figure S5. (A) Thermal ellipsoid (probability level 50\%) plot and (B) a packing diagram of bis(4bromophenyl)diazomethane $\left(6-\mathrm{N}_{2}\right)$. 
(A)

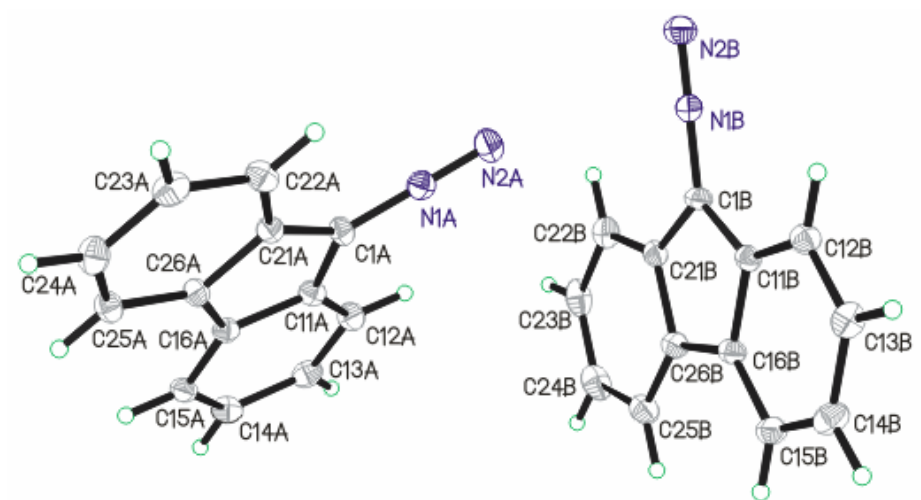

(B)

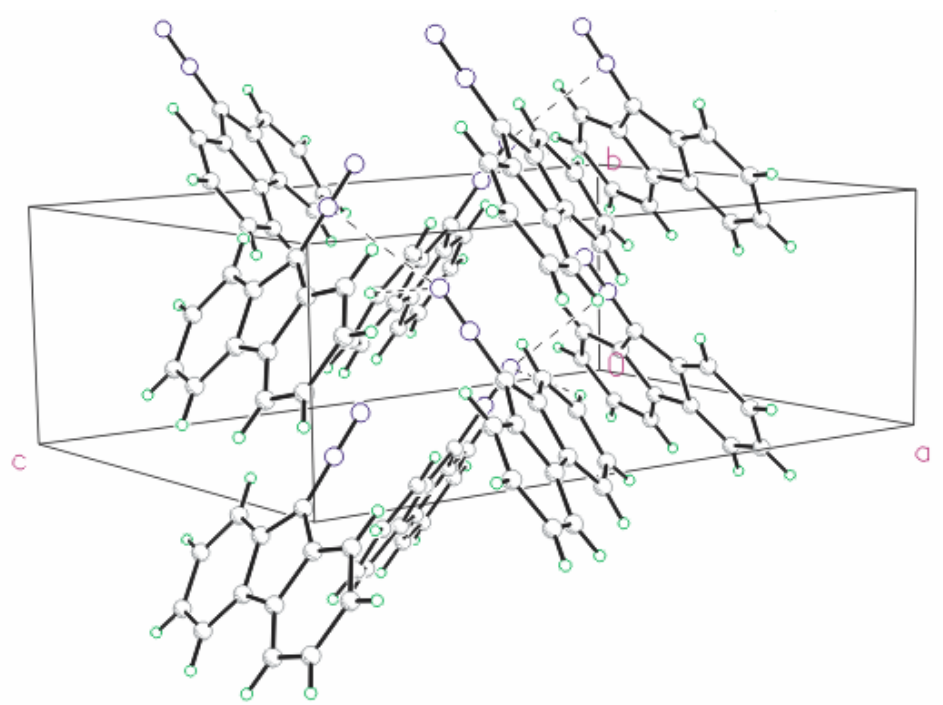

Figure S6. (A) Thermal ellipsoid (probability level 50\%) plot and (B) a packing diagram of diazofluorene $\left(7-\mathrm{N}_{2}\right)$. 
Table S1. Crystallographic Data for $2-\mathrm{N}_{2}-7-\mathrm{N}_{2}$

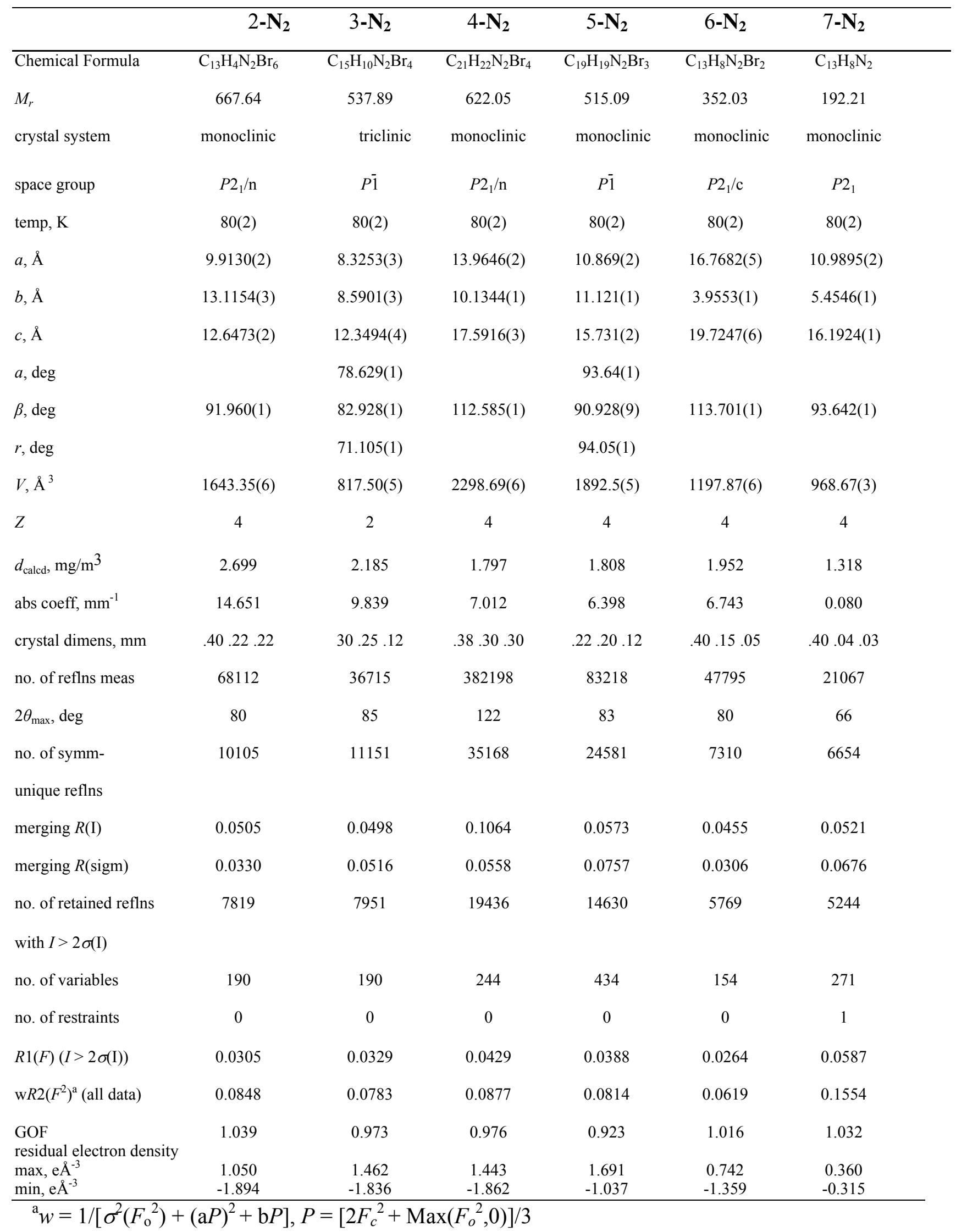




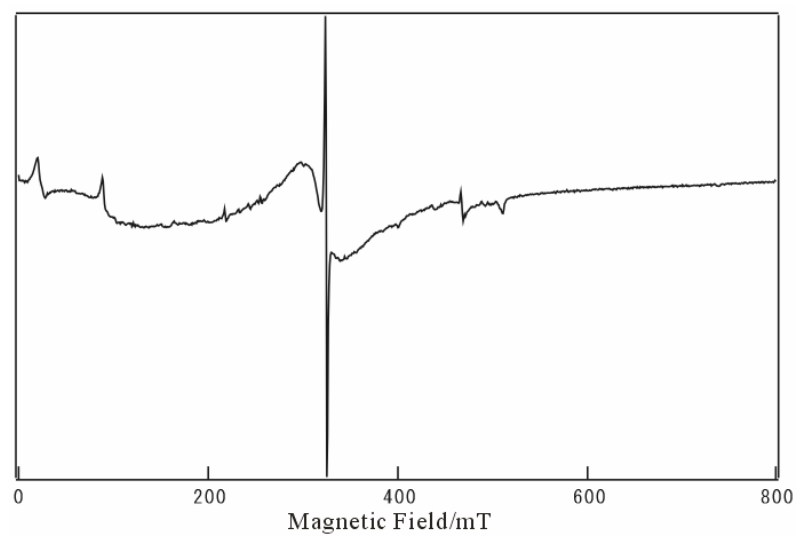

FigureS7. ESR spectrum of UV-irradiated microcrystals of bis(2,4,6-trichlorophenyl)diazomethane (1$\mathrm{N}_{2}$ ) at $20 \mathrm{~K}$.

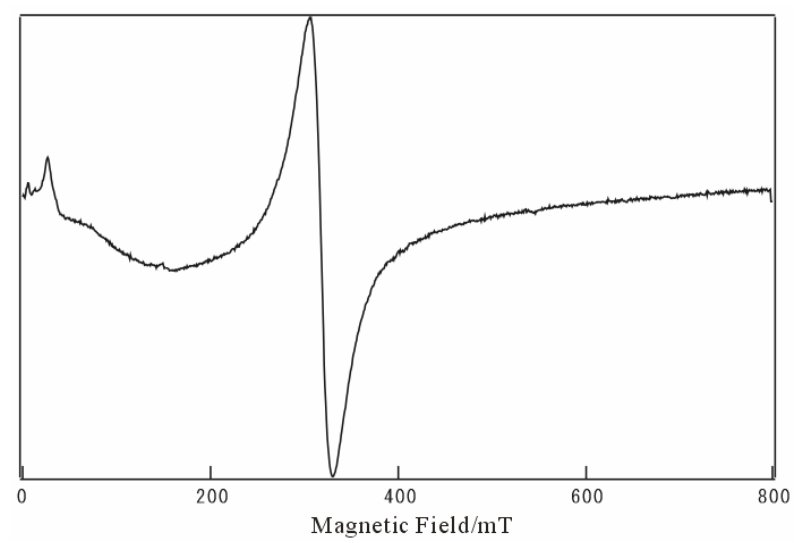

FigureS8. ESR spectrum of UV-irradiated microcrystals of bis(2,4,6-tribromophenyl)diazomethane (2$\mathrm{N}_{2}$ ) at $20 \mathrm{~K}$.

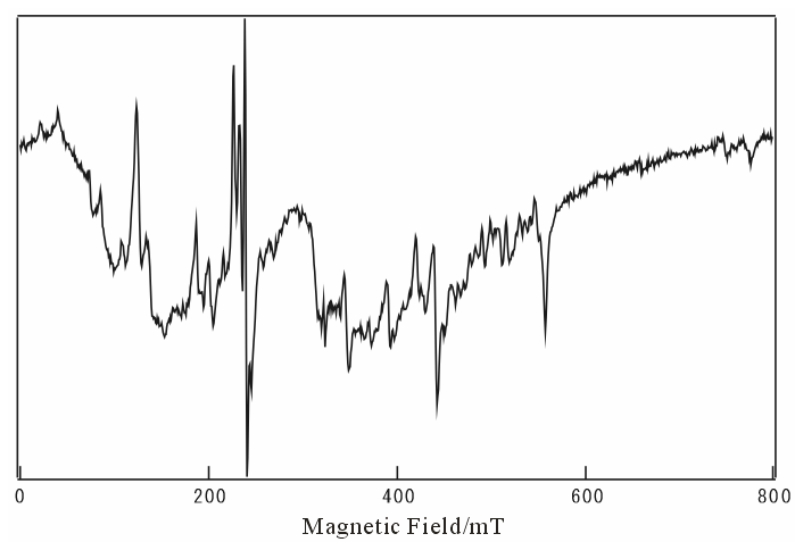

FigureS9. ESR spectrum of UV-irradiated microcrystals of bis(2,6-dibromo-4-tertbutylphenyl)diazomethane $\left(4-\mathrm{N}_{2}\right)$ at $20 \mathrm{~K}$. 


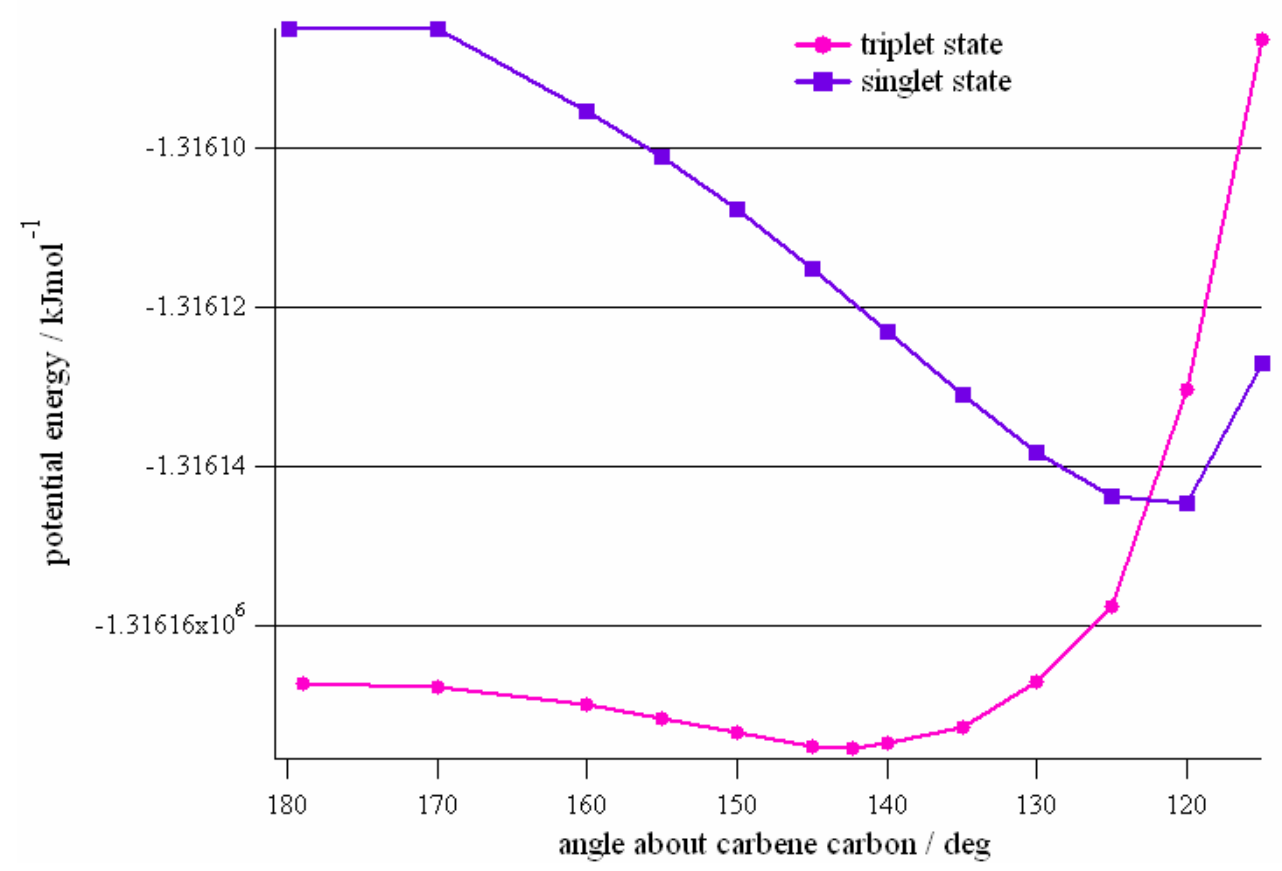

FigureS10. A potential energy surface of diphenyl carbene as a function of the carbene bond angle $(\theta)$ in the range of $115-179.9^{\circ}$ calculated at the UB3LYP/6-31G* levels of theory. 


\section{Cartesian coordinates for calculated molecules}

bis(2,4,6-trichlorophenyl)diazomethane $\left(\mathbf{1}-\mathrm{N}_{2}\right)$

Symmetry $\mathrm{C} 1$

singlet state

Zero-point correction $=0.137768$ (Hartree/Particle $)$

Sum of electronic and zero-point Energies $=-3368.265129$

$\mathrm{HF}=-3368.4028974$

Atomic Coordinates (Angstroms)

$\begin{array}{llll}\text { Type } & \text { X } & \text { Y } & \text { Z }\end{array}$

$$
\begin{array}{lrrr}
17 & -2.236798 & 1.931250 & 2.003791 \\
17 & -5.377236 & -1.643376 & -0.520293 \\
17 & -0.317258 & -0.996909 & -2.246446 \\
17 & 0.317116 & -1.001309 & 2.244357 \\
17 & 2.237391 & 1.935016 & -2.000190 \\
17 & 5.377248 & -1.644440 & 0.517594 \\
7 & -0.000232 & 2.418624 & 0.001119 \\
7 & -0.001088 & 3.558639 & 0.002418 \\
6 & -2.409990 & 0.750170 & 0.719881 \\
6 & -1.315294 & 0.437853 & -0.118674 \\
6 & -3.654519 & 0.137427 & 0.600150 \\
6 & 0.000053 & 1.105822 & 0.001017 \\
6 & -3.826434 & -0.846061 & -0.368392 \\
6 & -1.561710 & -0.553231 & -1.095393 \\
6 & -2.785647 & -1.205415 & -1.218901 \\
6 & 1.315403 & 0.437902 & 0.119610 \\
6 & 1.561653 & -0.555247 & 1.094291 \\
6 & 2.410317 & 0.751778 & -0.718156
\end{array}
$$




$$
\begin{array}{lrrr}
6 & 2.785619 & -1.207611 & 1.216798 \\
6 & 3.654784 & 0.138783 & -0.599417 \\
6 & 3.826540 & -0.846660 & 0.367176 \\
1 & -4.463507 & 0.410673 & 1.266047 \\
1 & -2.925794 & -1.961552 & -1.981275 \\
1 & 2.925511 & -1.965420 & 1.977556 \\
1 & 4.463869 & 0.413297 & -1.264676
\end{array}
$$

bis(2,4,6-tribromophenyl)diazomethane $\left(2-\mathrm{N}_{2}\right)$

Symmetry C1

singlet state

Zero-point correction $=0.135044($ Hartree $/$ Particle $)$

Sum of electronic and zero-point Energies $=-16037.331786$

$\mathrm{HF}=-16037.4668299$

Atomic Coordinates (Angstroms)

$\begin{array}{llll}\text { Type } & \mathrm{X} & \mathrm{Y} & \mathrm{Z}\end{array}$

$$
\begin{array}{llll}
35 & 2.107781 & 2.491107 & -1.593824 \\
35 & 0.272372 & -1.520350 & 2.125718 \\
35 & -0.206842 & -0.237729 & -2.608714 \\
35 & -2.244372 & 1.176510 & 2.601250 \\
35 & 5.576920 & -1.599613 & 0.180484 \\
35 & -5.455192 & -1.665297 & -1.077344 \\
7 & -0.086487 & 2.318021 & 0.636497 \\
7 & -0.127899 & 3.416854 & 0.938609 \\
6 & -0.039287 & 1.054612 & 0.289709 \\
6 & 1.298511 & 0.421885 & 0.249980 \\
6 & -1.329048 & 0.399194 & -0.024406
\end{array}
$$




$$
\begin{array}{llll}
6 & 2.369151 & 0.951057 & -0.503485 \\
6 & 1.591601 & -0.741664 & 0.992613 \\
6 & -1.554963 & -0.239686 & -1.262171 \\
6 & -2.419225 & 0.394318 & 0.873111 \\
6 & 3.636498 & 0.376430 & -0.523056 \\
6 & 2.839826 & -1.357667 & 0.968250 \\
6 & -2.756875 & -0.866592 & -1.579210 \\
6 & -3.643153 & -0.195999 & 0.572683 \\
6 & 3.856270 & -0.784520 & 0.210777 \\
6 & -3.795946 & -0.832060 & -0.654792 \\
1 & 4.427116 & 0.818052 & -1.115839 \\
1 & 3.017785 & -2.252867 & 1.550204 \\
1 & -2.883507 & -1.349442 & -2.539703 \\
\hline 1 & -4.451036 & -0.174558 & 1.292659
\end{array}
$$

bis(2,6-dibromo-4-methylphenyl)diazomethane $\left(3-\mathrm{N}_{2}\right)$

Symmetry C1

singlet state

Zero-point correction $=0.210661$ (Hartree/Particle)

Sum of electronic and zero-point Energies $=-10973.689461$

$\mathrm{HF}=-10973.900122$

\begin{tabular}{lllc} 
Atomic & \multicolumn{3}{c}{ Coordinates (Angstroms) } \\
Type & X & Y & Z
\end{tabular}

$$
\begin{array}{llll}
35 & 0.234951 & -0.793980 & -2.400550 \\
35 & 2.091303 & 2.557167 & 1.915782 \\
35 & -2.348053 & 1.882006 & -2.351075 \\
35 & -0.169628 & -0.335717 & 2.511339
\end{array}
$$




$$
\begin{aligned}
& \begin{array}{lllll}
7 & -0.157436 & 2.721769 & -0.266867
\end{array} \\
& \begin{array}{llll}
7 & -0.223084 & 3.855746 & -0.378035
\end{array} \\
& \begin{array}{llll}
6 & -0.082156 & 1.420417 & -0.139278
\end{array} \\
& \begin{array}{llll}
6 & 1.266277 & 0.815006 & -0.234072
\end{array} \\
& \begin{array}{llll}
6 & -1.353608 & 0.694853 & 0.086010
\end{array} \\
& \begin{array}{llll}
6 & 1.566424 & -0.197378 & -1.168838
\end{array} \\
& \begin{array}{llll}
6 & 2.347684 & 1.222755 & 0.574880
\end{array} \\
& \begin{array}{llll}
6 & -2.465367 & 0.812056 & -0.774424
\end{array} \\
& \begin{array}{llll}
6 & -1.546634 & -0.144924 & 1.202399
\end{array} \\
& \begin{array}{llll}
6 & 2.825274 & -0.780525 & -1.267219
\end{array} \\
& \begin{array}{llll}
6 & 3.620204 & 0.671943 & 0.466411
\end{array} \\
& 6 \begin{array}{llll}
6 & -3.667798 & 0.151006 & -0.547132
\end{array} \\
& \begin{array}{llll}
6 & -2.731671 & -0.837860 & 1.425900
\end{array} \\
& 6 \quad 3.875576 \quad-0.351733 \quad-0.450024 \\
& \begin{array}{llll}
6 & -3.814847 & -0.698206 & 0.552949
\end{array} \\
& \begin{array}{llll}
6 & 5.239487 & -0.992971 & -0.539462
\end{array} \\
& \begin{array}{llll}
6 & -5.097507 & -1.461496 & 0.780130
\end{array} \\
& \begin{array}{llll}
1 & 2.989924 & -1.558752 & -2.004937
\end{array} \\
& 1 \quad 4.409519 \quad 1.033965 \quad 1.116647 \\
& 1 \quad-4.485958 \quad 0.287729 \quad-1.246277 \\
& 1 \quad-2.814631 \quad-1.472139 \quad 2.302140 \\
& 1 \quad 5.454201 \quad-1.333175 \quad-1.557663 \\
& 1 \quad 6.028575 \quad-0.296670 \quad-0.238571 \\
& 1 \quad 5.305836 \quad-1.868960 \quad 0.118983 \\
& 1 \quad-5.055382 \quad-2.450759 \quad 0.305900 \\
& 1 \quad-5.284142 \quad-1.619013 \quad 1.847341 \\
& \begin{array}{llll}
1 & -5.957333 & -0.932159 & 0.357613
\end{array}
\end{aligned}
$$

bis(2,6-dibromo-4-tert-butylphenyl)diazomethane (4- $\left.\mathrm{N}_{2}\right)$ 


\section{Symmetry C1}

singlet state

Zero-point correction $=0.381446($ Hartree $/$ Particle $)$

Sum of electronic and zero-point Energies $=-11209.393881$

$\mathrm{HF}=-11209.7753272$

\section{Atomic Coordinates (Angstroms)}

$\begin{array}{llll}\text { Type } & X & Y & Z\end{array}$

$$
\begin{array}{lrrr}
35 & -0.073363 & 0.612834 & 2.415218 \\
35 & -2.528918 & 2.038583 & -2.606847 \\
35 & 2.110108 & 3.405187 & 1.271276 \\
35 & 0.100909 & -0.591478 & -2.372965 \\
7 & -0.249233 & 3.213665 & -0.792052 \\
7 & -0.338996 & 4.316413 & -1.071306 \\
6 & -0.145464 & 1.947809 & -0.471809 \\
6 & -1.390699 & 1.253820 & -0.071802 \\
6 & 1.207000 & 1.348497 & -0.539797 \\
6 & -1.515092 & 0.591022 & 1.162251 \\
6 & -2.548347 & 1.222362 & -0.879863 \\
6 & 2.319434 & 1.881930 & 0.136818 \\
6 & 1.481042 & 0.203704 & -1.317202 \\
6 & -2.675059 & -0.078531 & 1.550175 \\
6 & -3.721259 & 0.589540 & -0.492543 \\
6 & 3.595792 & 1.329941 & 0.054757 \\
6 & 2.740414 & -0.376296 & -1.382624 \\
6 & 3.833561 & 0.178170 & -0.700747 \\
6 & -3.809064 & -0.088479 & 0.732141 \\
6 & -5.115943 & -0.797924 & 1.125956 \\
6 & 5.219161 & -0.482316 & -0.801474
\end{array}
$$




$$
\begin{aligned}
& \begin{array}{llll}
6 & -5.455283 & -1.869243 & 0.061680
\end{array} \\
& \begin{array}{llll}
6 & -6.259359 & 0.240898 & 1.194924
\end{array} \\
& \begin{array}{llll}
6 & -5.009084 & -1.493499 & 2.496209
\end{array} \\
& \begin{array}{llll}
6 & 6.285110 & 0.277873 & 0.009662
\end{array} \\
& \begin{array}{llll}
6 & 5.133710 & -1.928916 & -0.259815
\end{array} \\
& \begin{array}{lllll}
6 & 5.665237 & -0.511691 & -2.282647
\end{array} \\
& \begin{array}{llll}
1 & -2.679384 & -0.569733 & 2.513408
\end{array} \\
& \begin{array}{llll}
1 & -4.565180 & 0.614332 & -1.170857
\end{array} \\
& \begin{array}{llll}
1 & 4.392889 & 1.807530 & 0.609321
\end{array} \\
& \begin{array}{llll}
1 & 2.865849 & -1.260657 & -1.997523
\end{array} \\
& 1 \quad-5.590974 \quad-1.431051 \quad-0.931989 \\
& \begin{array}{llll}
1 & -6.386480 & -2.383439 & 0.328050
\end{array} \\
& \begin{array}{llll}
1 & -4.660057 & -2.619937 & -0.008405
\end{array} \\
& \begin{array}{llll}
1 & -6.048751 & 1.008579 & 1.947087
\end{array} \\
& \begin{array}{llll}
1 & -7.200848 & -0.251533 & 1.465800
\end{array} \\
& \begin{array}{llll}
1 & -6.411992 & 0.745492 & 0.234631
\end{array} \\
& \begin{array}{llll}
1 & -4.801129 & -0.780848 & 3.301761
\end{array} \\
& \begin{array}{llll}
1 & -4.226551 & -2.261475 & 2.505057
\end{array} \\
& \begin{array}{llll}
1 & -5.958353 & -1.987709 & 2.731261
\end{array} \\
& \begin{array}{llll}
1 & 6.412328 & 1.306478 & -0.345802
\end{array} \\
& \begin{array}{llll}
1 & 7.252059 & -0.228027 & -0.091793
\end{array} \\
& \begin{array}{llll}
1 & 6.041112 & 0.310616 & 1.076962
\end{array} \\
& 1 \quad 4.421464 \quad-2.536954 \quad-0.827104 \\
& 1 \quad 4.819906 \quad-1.937923 \quad 0.789798 \\
& 1 \quad 6.113896 \quad-2.416301 \quad-0.325060 \\
& 1 \quad 5.738166 \quad 0.501758 \quad-2.691110 \\
& 1 \quad 4.966202 \quad-1.076355 \quad-2.909481 \\
& 1 \quad 6.649520 \quad-0.986716 \quad-2.371589
\end{aligned}
$$


(2,4,6-triboromophenyl)(2,6-dimethyl-4-tert-butylphenyl)diazomethane $\left(5-\mathrm{N}_{2}\right)$

Symmetry C1

singlet state

Zero-point correction $=0.334957$ (Hartree/Particle $)$

Sum of electronic and zero-point Energies $=-8559.713342$

$\mathrm{HF}=-8560.0482992$

Atomic

Coordinates (Angstroms)

Type $\quad \mathrm{X} \quad \mathrm{Y} \quad \mathrm{Z}$
$35 \mathrm{Br} \quad-4.005119 \quad 3.055798 \quad-0.103402$
$35 \mathrm{Br} \quad-2.080724 \quad-2.165208 \quad 1.465088$
$35 \mathrm{Br} \quad-7.285602 \quad-1.513888 \quad-0.624603$
$\begin{array}{llll}7 \mathrm{~N} & -1.898044 & 1.994514 & 1.897441\end{array}$
$\begin{array}{llll}7 \mathrm{~N} & -1.916378 & 2.870352 & 2.632653\end{array}$
6 C $\quad 1.050444 \quad 0.152729 \quad-1.241047$
$\begin{array}{llll}6 \mathrm{C} & 2.093568 & -0.130718 & -0.355796\end{array}$
6 C $\quad-0.229771 \quad 0.519273 \quad-0.808088$
6 C $\quad 1.805115 \quad-0.045415 \quad 1.013179$
6 C $\quad-0.493984 \quad 0.584516 \quad 0.577151$
6 C $\quad-1.280624 \quad 0.837770 \quad-1.847640$
$\begin{array}{llll}6 \mathrm{C} & 3.506574 & -0.523724 & -0.822453\end{array}$
$\begin{array}{llll}6 \mathrm{C} & 0.542259 & 0.294068 & 1.499211\end{array}$
6 C $\quad-1.845916 \quad 0.987178 \quad 1.061367$
$\begin{array}{llll}6 \mathrm{C} & 3.633602 & -0.548703 & -2.357598\end{array}$
6 C $\quad 3.848852 \quad-1.935178 \quad-0.288295$
$\begin{array}{llll}6 \mathrm{C} & 4.532706 & 0.494840 & -0.271266\end{array}$
$\begin{array}{llll}6 \mathrm{C} & 0.307747 & 0.305929 & 2.992274\end{array}$
6 C $\quad-3.143653 \quad 0.401590 \quad 0.660384$
6 C $\quad-3.397059 \quad-0.985251 \quad 0.751180$ 

6 C $\quad-4.602503 \quad-1.563382 \quad 0.363655$
$\begin{array}{llll}6 \mathrm{C} & -5.444621 & 0.633005 & -0.204245\end{array}$
6 C $\quad-5.623192 \quad-0.742247 \quad-0.105091$
$\begin{array}{llll}6 \mathrm{C} & -4.216022 & 1.176056 & 0.161650\end{array}$
$\begin{array}{llll}1 \mathrm{H} & 2.583672 & -0.265192 & 1.739167\end{array}$
$\begin{array}{llll}1 \mathrm{H} & 1.222459 & 0.110223 & -2.311197\end{array}$
$1 \mathrm{H} \quad-1.867673 \quad 1.722015 \quad-1.580500$
$1 \mathrm{H} \quad-1.988501 \quad 0.009156 \quad-1.979378$
$1 \mathrm{H} \quad-0.812774 \quad 1.023019 \quad-2.819407$
$1 \mathrm{H} \quad 4.654189-0.832257 \quad-2.638415$
$\begin{array}{llll}1 \mathrm{H} & 3.430268 & 0.433118 & -2.800107\end{array}$
$1 \mathrm{H} \quad 2.952343 \quad-1.276859 \quad-2.812246$
$\begin{array}{llll}1 \mathrm{H} & 4.856997 & -2.229019 & -0.605584\end{array}$
$\begin{array}{llll}1 \mathrm{H} & 3.141622 & -2.680475 & -0.669770\end{array}$
$\begin{array}{llll}1 \mathrm{H} & 3.818835 & -1.975498 & 0.805519\end{array}$
$1 \mathrm{H} \quad 5.546666 \quad 0.225945 \quad-0.591784$
$\begin{array}{llll}1 \mathrm{H} & 4.527309 & 0.527976 & 0.823137\end{array}$
$\begin{array}{llll}1 \mathrm{H} & 4.317157 & 1.505191 & -0.637137\end{array}$
$1 \mathrm{H} \quad 0.167756 \quad 1.317364 \quad 3.394641$
$\begin{array}{llll}1 \mathrm{H} & 1.159578 & -0.136416 & 3.517428\end{array}$
$1 \mathrm{H} \quad-0.587585 \quad-0.270991 \quad 3.249376$
$1 \mathrm{H} \quad-4.746165 \quad-2.632733 \quad 0.450440$
$1 \mathrm{H} \quad-6.233830 \quad 1.271538 \quad-0.579902$

bis(4-bromophenyl)diazomethane $\left(6-\mathrm{N}_{2}\right)$

Symmetry C1

singlet state

Zero-point correction $=0.176242($ Hartree/Particle $)$

Sum of electronic and zero-point Energies $=\mathbf{- 5 7 5 2 . 8 8 9 1 0 0}$ 


$$
H F=-5753.0653413
$$

\begin{tabular}{llcc} 
Atomic & & \multicolumn{3}{c}{ Coordinates (Angstroms) } \\
Type & X & Y & Z
\end{tabular}

$$
\begin{array}{lrrr}
35 & 5.541218 & -1.659127 & 0.044840 \\
35 & -5.541195 & -1.659187 & -0.044927 \\
7 & 0.000038 & 2.415789 & 0.000000 \\
7 & 0.000019 & 3.562258 & 0.000421 \\
6 & -0.000004 & 1.104976 & 0.000076 \\
6 & 1.319067 & 0.440696 & -0.008301 \\
6 & -1.319076 & 0.440728 & 0.008294 \\
6 & 2.442416 & 1.058573 & -0.587466 \\
6 & 1.492608 & -0.818501 & 0.594773 \\
6 & -1.492430 & -0.818808 & -0.594130 \\
6 & -2.442621 & 1.058913 & 0.586769 \\
6 & 3.692729 & 0.447084 & -0.567017 \\
6 & 2.736882 & -1.443759 & 0.606567 \\
6 & -2.736694 & -1.444086 & -0.605945 \\
6 & -3.692910 & 0.447392 & 0.566291 \\
6 & 3.831951 & -0.806281 & 0.026302 \\
6 & -3.831944 & -0.806308 & -0.026365 \\
1 & 2.341025 & 2.027737 & -1.068195 \\
1 & 0.650259 & -1.309479 & 1.070774 \\
1 & -0.649942 & -1.310055 & -1.069606 \\
1 & -2.341412 & 2.028357 & 1.066971 \\
1 & 4.549159 & 0.935847 & -1.018595 \\
1 & 2.856326 & -2.414284 & 1.075862 \\
1 & -2.855985 & -2.414877 & -1.074736 \\
1 & -4.549485 & 0.936402 & 1.017334
\end{array}
$$


diazofluorene $\left(7-\mathrm{N}_{2}\right)$,

Symmetry C1

singlet state

Zero-point correction $=0.174998($ Hartree/Particle $)$

Sum of electronic and zero-point Energies $=-609.494902$

$\mathrm{HF}=-609.6699003$

Atomic Coordinates (Angstroms)

$\begin{array}{llll}\text { Type } & \mathrm{X} & \mathrm{Y} & \mathrm{Z}\end{array}$

$\begin{array}{lllll}7 & \mathrm{~N} & -0.000582 & 2.904381 & -0.000297\end{array}$

$\begin{array}{lllll}7 & \mathrm{~N} & -0.001552 & 4.049139 & 0.000193\end{array}$

$\begin{array}{lllll}6 & \mathrm{C} & -0.000181 & 1.601597 & 0.000002\end{array}$

$\begin{array}{lllll}6 & C & -1.188846 & 0.752528 & 0.000052\end{array}$

$\begin{array}{lllll}6 & \mathrm{C} & 1.188518 & 0.753073 & -0.000260\end{array}$

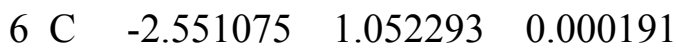

6 C $\quad-0.731474 \quad-0.592588 \quad-0.000100$

$\begin{array}{lllll}6 & \mathrm{C} & 2.550890 & 1.053035 & -0.000316\end{array}$

6 C $\quad 0.731766 \quad-0.592355 \quad-0.000093$

$\begin{array}{lllll}6 & \mathrm{C} & -3.463274 & -0.003797 & 0.000170\end{array}$

$\begin{array}{lllll}6 & \mathrm{C} & -1.659978 & -1.635371 & -0.000276\end{array}$

$\begin{array}{lllll}6 \text { C } & 3.463390 & -0.002695 & 0.000149\end{array}$

$\begin{array}{lllll}6 \text { C } & 1.660628 & -1.634813 & -0.000042\end{array}$

$\begin{array}{lllll}6 & C & -3.022825 & -1.335125 & -0.000094\end{array}$

$\begin{array}{lllll}6 & C & 3.023398 & -1.334305 & 0.000344\end{array}$

$\begin{array}{lllll}1 \mathrm{H} & 2.899081 & 2.082788 & -0.000578\end{array}$

$\begin{array}{lllll}1 & \mathrm{H} & -2.899624 & 2.081927 & 0.000319\end{array}$

$\begin{array}{lllll}1 \mathrm{H} & 4.528566 & 0.211546 & 0.000243\end{array}$ 
$1 \mathrm{H} \quad 1.327894 \quad-2.669711 \quad-0.000154$

$1 \mathrm{H} \quad-1.327133 \quad-2.670191 \quad-0.000381$

$\begin{array}{lllll}1 & \mathrm{H} & -4.528548 & 0.209873 & 0.000366\end{array}$

$\begin{array}{lllll}1 & \mathrm{H} & 3.752128 & -2.140014 & 0.000673\end{array}$

$1 \mathrm{H} \quad-3.751166 \quad-2.141216 \quad-0.000117$

bis(2,4,6-trichlorophenyl)carbene (1)

Symmetry $\mathrm{C} 1$

triplet state

Zero-point correction $=0.125195($ Hartree/Particle $)$

Sum of electronic and zero-point Energies $=-3258.739011$

$\mathrm{HF}=-3258.8642065$

Atomic Coordinates (Angstroms)

$\begin{array}{llll}\text { Type } & \mathrm{X} & \mathrm{Y} & \mathrm{Z}\end{array}$
$17 \mathrm{Cl} \quad 1.606786 \quad 2.273726 \quad-1.762305$
$17 \mathrm{Cl} \quad 0.954856 \quad-1.818139 \quad 1.829038$
$17 \mathrm{Cl} \quad-0.932025 \quad-1.828021 \quad-1.799841$
$17 \mathrm{Cl} \quad-1.631917 \quad 2.278790 \quad 1.766209$
$\begin{array}{llll}17 \mathrm{Cl} & 5.893663 & -0.424123 & 0.134863\end{array}$
$17 \mathrm{Cl} \quad-5.891824 \quad-0.436904 \quad-0.166230$
$\begin{array}{lllll}6 & \mathrm{C} & -0.000930 & 0.454864 & 0.016342\end{array}$
$\begin{array}{lllll}6 & \mathrm{C} & 1.353637 & 0.216695 & 0.027734\end{array}$
$\begin{array}{lllll}6 & C & -1.354330 & 0.214602 & -0.011909\end{array}$
$\begin{array}{lllll}6 \mathrm{C} & 2.264121 & 1.016642 & -0.743784\end{array}$
$\begin{array}{lllll}6 & \mathrm{C} & 1.969647 & -0.795791 & 0.842291\end{array}$

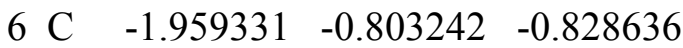
$\begin{array}{lllll}6 & C & -2.275422 & 1.015690 & 0.746197\end{array}$ 

$\begin{array}{lllll}6 & C & 3.634104 & 0.828908 & -0.718117\end{array}$
$\begin{array}{lllll}6 \mathrm{C} & 3.338838 & -0.994176 & 0.876004\end{array}$
$\begin{array}{lllll}6 & C & -3.327626 & -1.004579 & -0.876554\end{array}$
$\begin{array}{lllll}6 \mathrm{C} & -3.644492 & 0.824900 & 0.706114\end{array}$
$\begin{array}{lllll}6 & \mathrm{C} & 4.163831 & -0.178937 & 0.094464\end{array}$
6 C $\quad-4.163078 \quad-0.187738 \quad-0.107840$
$\begin{array}{lllll}1 \mathrm{H} & 4.284189 & 1.452465 & -1.319667\end{array}$
$\begin{array}{lllll}1 \mathrm{H} & 3.763192 & -1.770727 & 1.500837\end{array}$

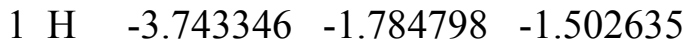
$\begin{array}{lllll}1 & \mathrm{H} & -4.302539 & 1.449898 & 1.297424\end{array}$

bis(2,4,6-trichlorophenyl)carbene (1)

Symmetry C1

singlet state

Zero-point correction $=0.126078$ (Hartree/Particle)

Sum of electronic and zero-point Energies $=-3258.717804$

$\mathrm{HF}=-3258.8438813$
Atomic
Coordinates (Angstroms)
Type
X $\quad$ Y $\quad$ Z
$\begin{array}{llll}17 & 1.909077 & 2.312226 & -1.876435\end{array}$
$\begin{array}{llll}17 & 5.677134 & -0.824461 & 0.375942\end{array}$
$\begin{array}{llll}17 & 0.550552 & -1.498404 & 1.852391\end{array}$
$\begin{array}{lllll}17 & -0.550363 & -1.498627 & -1.852002\end{array}$
$\begin{array}{llll}17 & -1.909250 & 2.312333 & 1.876341\end{array}$
$\begin{array}{llll}17 & -5.677112 & -0.824572 & -0.376099\end{array}$
$\begin{array}{llll}6 & 2.331329 & 1.045563 & -0.756195\end{array}$
$\begin{array}{llll}6 & 1.279186 & 0.425484 & -0.000042\end{array}$ 


$$
\begin{array}{lrrr}
6 & 3.654398 & 0.650150 & -0.683082 \\
6 & -0.000014 & 1.002453 & 0.000023 \\
6 & 4.003123 & -0.352897 & 0.226307 \\
6 & 1.708240 & -0.636581 & 0.864659 \\
6 & 3.042606 & -0.985612 & 1.016253 \\
6 & -1.279209 & 0.425482 & 0.000117 \\
6 & -1.708168 & -0.636665 & -0.864523 \\
6 & -2.331422 & 1.045566 & 0.756171 \\
6 & -3.042527 & -0.985719 & -1.016214 \\
6 & -3.654475 & 0.650141 & 0.682973 \\
6 & -4.003117 & -0.352962 & -0.226402 \\
\hline 1 & 3.328667 & -1.762586 & 1.714653 \\
\hline 1 & 4.407307 & 1.126183 & -1.299162 \\
\hline 1 & -3.328520 & -1.762706 & -1.714627 \\
\hline 1 & -4.407443 & 1.126215 & 1.298947
\end{array}
$$

bis(2,4,6-tribromophenyl)carbene (2)

Symmetry C1

triplet state

Zero-point correction $=0.122832($ Hartree/Particle $)$

Sum of electronic and zero-point Energies $=-15927.801498$

$\mathrm{HF}=-15927.9243299$

\begin{tabular}{lrcc} 
Atomic & \multicolumn{3}{c}{ Coordinates (Angstroms) } \\
Type & $\mathrm{X}$ & $\mathrm{Y}$ & $\mathrm{Z}$ \\
----------------------------------------------- & & \\
35 & -1.606389 & 2.598435 & 1.574236 \\
35 & -0.786070 & -2.056929 & -1.677857 \\
35 & 0.790072 & -2.055838 & 1.682721
\end{tabular}




$$
\begin{array}{lrrr}
35 & 1.601864 & 2.598611 & -1.572485 \\
35 & -6.026531 & -0.512531 & -0.173785 \\
35 & 6.026932 & -0.509824 & 0.167495 \\
6 & -0.000220 & 0.512387 & 0.003104 \\
6 & -1.351493 & 0.237961 & -0.029623 \\
6 & 1.351339 & 0.238471 & 0.032579 \\
6 & -2.290089 & 1.100183 & 0.626417 \\
6 & -1.929494 & -0.860622 & -0.748442 \\
6 & 1.931274 & -0.859511 & 0.750640 \\
6 & 2.288124 & 1.100966 & -0.625572 \\
6 & -3.655729 & 0.889718 & 0.592299 \\
6 & -3.294654 & -1.085448 & -0.790223 \\
6 & 3.296611 & -1.083757 & 0.789736 \\
6 & 3.653956 & 0.891119 & -0.594176 \\
6 & -4.150939 & -0.208973 & -0.117502 \\
6 & 4.151097 & -0.207107 & 0.114959 \\
1 & -4.329011 & 1.564765 & 1.105602 \\
1 & -3.691756 & -1.930211 & -1.339217 \\
1 & 3.695225 & -1.928145 & 1.338209 \\
1 & 4.325876 & 1.566284 & -1.109110
\end{array}
$$

bis(2,4,6-tribromophenyl)carbene (2)

Symmetry C1

singlet state

Zero-point correction $=0.123061$ (Hartree/Particle $)$

Sum of electronic and zero-point Energies $=-15927.781231$

$\mathrm{HF}=-15927.9042918$

Atomic Coordinates (Angstroms) 
$\begin{array}{llll}\text { Type } & \mathrm{X} & \mathrm{Y} & \mathrm{Z}\end{array}$
$\begin{array}{llll}35 & 0.452326 & -1.656341 & 1.859359\end{array}$
$\begin{array}{llll}35 & -0.452269 & -1.656404 & -1.859243\end{array}$
$\begin{array}{llll}35 & -1.831540 & 2.381730 & 2.004260\end{array}$
$\begin{array}{llll}35 & -5.839868 & -0.793963 & -0.444570\end{array}$
$\begin{array}{llll}6 & 2.326977 & 1.039258 & -0.759067\end{array}$
$\begin{array}{llll}6 & 1.281014 & 0.414285 & -0.003746\end{array}$
$\begin{array}{llll}6 & 3.655183 & 0.670476 & -0.670035\end{array}$
$\begin{array}{llll}6 & -0.000004 & 0.988681 & 0.000024\end{array}$
$\begin{array}{llll}6 & 4.011246 & -0.321076 & 0.249598\end{array}$
$\begin{array}{llll}6 & 1.712424 & -0.650696 & 0.852075\end{array}$
$\begin{array}{llll}6 & 3.050385 & -0.976941 & 1.019852\end{array}$
$\begin{array}{llll}6 & -1.281021 & 0.414286 & 0.003765\end{array}$
$\begin{array}{llll}6 & -1.712401 & -0.650722 & -0.852038\end{array}$
$\begin{array}{llll}6 & -2.327007 & 1.039267 & 0.759050\end{array}$
$\begin{array}{llll}6 & -3.050354 & -0.976982 & -1.019840\end{array}$
$\begin{array}{llll}6 & -3.655206 & 0.670472 & 0.669994\end{array}$
$\begin{array}{llll}6 & -4.011240 & -0.321104 & -0.249626\end{array}$
$\begin{array}{llll}1 & 3.339983 & -1.755128 & 1.714718\end{array}$
$1 \quad 4.405641 \quad 1.154152 \quad-1.282560$
$1 \quad-3.339931 \quad-1.755193 \quad-1.714689$
$1 \quad-4.405684 \quad 1.154153 \quad 1.282492$

bis(2,6-dibromo-4-tert-butylphenyl)carbene (4)

Symmetry $\mathrm{C} 1$

triplet state

Zero-point correction $=0.369355$ (Hartree/Particle $)$

Sum of electronic and zero-point Energies $=-11099.862493$ 


$$
H F=-11100.2318477
$$

\begin{tabular}{llcc} 
Atomic & & \multicolumn{3}{c}{ Coordinates (Angstroms) } \\
Type & X & Y & Z
\end{tabular}

$$
\begin{array}{lrrr}
35 & 0.717505 & -1.682972 & -1.701810 \\
35 & 1.700950 & 2.911256 & 1.599862 \\
35 & -1.645481 & 2.946997 & -1.586787 \\
35 & -0.774302 & -1.677079 & 1.702322 \\
6 & 0.001921 & 0.897716 & 0.011360 \\
6 & 1.346958 & 0.583064 & -0.035852 \\
6 & -1.349470 & 0.606151 & 0.038253 \\
6 & 1.895600 & -0.517227 & -0.765371 \\
6 & 2.323918 & 1.402062 & 0.615690 \\
6 & -2.302442 & 1.442127 & -0.619081 \\
6 & -1.925487 & -0.491185 & 0.757051 \\
6 & 3.256257 & -0.776244 & -0.820065 \\
\hline 6 & 3.677991 & 1.146590 & 0.559630 \\
6 & -3.666795 & 1.209746 & -0.584508 \\
\hline 6 & -3.286025 & -0.725356 & 0.790079 \\
6 & 4.184253 & 0.044954 & -0.160959 \\
6 & -4.196567 & 0.116601 & 0.120213 \\
6 & 5.699161 & -0.211866 & -0.200015 \\
6 & -5.702847 & -0.182585 & 0.185138 \\
6 & 6.217957 & -0.428633 & 1.242063 \\
6 & 6.410566 & 1.013168 & -0.823413 \\
6 & 6.058697 & -1.455609 & -1.034455 \\
6 & -6.534894 & 0.842457 & -0.607997 \\
\hline 6.970737 & -1.587672 & -0.406526 \\
\hline 6 & -6.168737 & -0.149094 & 1.660605
\end{array}
$$




$$
\begin{array}{lrrr}
1 & 3.586054 & -1.634482 & -1.390515 \\
1 & 4.351307 & 1.814447 & 1.084824 \\
1 & -4.315618 & 1.893023 & -1.116696 \\
1 & -3.644078 & -1.581599 & 1.350428 \\
1 & 6.028081 & 0.440830 & 1.879844 \\
1 & 7.300375 & -0.604111 & 1.231706 \\
1 & 5.736749 & -1.296711 & 1.706340 \\
1 & 6.067532 & 1.186090 & -1.849625 \\
1 & 7.494275 & 0.847627 & -0.849838 \\
1 & 6.228117 & 1.928132 & -0.250307 \\
1 & 5.743433 & -1.352942 & -2.078974 \\
1 & 5.606535 & -2.366571 & -0.626157 \\
\hline 1 & 7.144954 & -1.597154 & -1.030902 \\
\hline 1 & -6.412309 & 1.858640 & -0.216529 \\
\hline 1 & -7.597721 & 0.587381 & -0.534753 \\
\hline 1 & -6.270177 & 0.848583 & -1.671370 \\
1 & -5.439636 & -2.371476 & 0.143534 \\
1 & -5.653913 & -1.639790 & -1.454214 \\
1 & -7.041910 & -1.818031 & -0.362893 \\
1 & -5.998459 & 0.838341 & 2.104075 \\
1 & -5.640589 & -0.887146 & 2.273161 \\
1 & -7.240961 & -0.370453 & 1.723418
\end{array}
$$

bis(2,6-dibromo-4-tert-butylphenyl)carbene (4)

\section{Symmetry C1}

singlet state

Zero-point correction $=0.369545($ Hartree/Particle $)$

Sum of electronic and zero-point Energies $=-11099.842556$

$\mathrm{HF}=-11100.2121002$ 


\begin{tabular}{llcc} 
Atomic & & \multicolumn{2}{c}{ Coordinates (Angstroms) } \\
Type & $\mathrm{X}$ & $\mathrm{Y}$ & $\mathrm{Z}$
\end{tabular}

$\begin{array}{llll}35 & 0.388597 & 2.230361 & -0.772063\end{array}$

$\begin{array}{llll}35 & 1.818831 & -2.103236 & 2.701929\end{array}$

$\begin{array}{llll}35 & -1.847713 & 2.293820 & 2.623727\end{array}$

$\begin{array}{llll}35 & -0.397548 & -2.165313 & -0.684022\end{array}$

$\begin{array}{llll}6 & -0.005887 & 0.083159 & 1.611440\end{array}$

$\begin{array}{llll}6 & 1.260919 & 0.099684 & 0.989860\end{array}$

$\begin{array}{llll}6 & -1.277095 & 0.028557 & 1.002056\end{array}$

$\begin{array}{llll}6 & 1.665543 & 1.036290 & -0.001832\end{array}$

$\begin{array}{llll}6 & 2.312170 & -0.729594 & 1.479795\end{array}$

$\begin{array}{llll}6 & -2.331265 & 0.866266 & 1.462508\end{array}$

$\begin{array}{llll}6 & -1.679467 & -0.951840 & 0.046471\end{array}$

$\begin{array}{lllll}6 & 2.992275 & 1.186800 & -0.388097\end{array}$

$\begin{array}{llll}6 & 3.614262 & -0.643879 & 1.037479\end{array}$

$\begin{array}{llll}6 & -3.640640 & 0.754446 & 1.032162\end{array}$

$\begin{array}{llll}6 & -3.002638 & -1.122661 & -0.324452\end{array}$

$\begin{array}{llll}6 & 3.996755 & 0.344976 & 0.107806\end{array}$

$\begin{array}{llll}6 & -4.016491 & -0.269527 & 0.146847\end{array}$

$\begin{array}{llll}6 & 5.466691 & 0.458110 & -0.325029\end{array}$

$6 \begin{array}{llll}6 & -5.467191 & -0.480453 & -0.314543\end{array}$

$\begin{array}{llll}6 & 5.906817 & -0.868559 & -0.990840\end{array}$

$\begin{array}{llll}6 & 6.346718 & 0.724577 & 0.920100\end{array}$

$\begin{array}{lllll}6 & 5.693503 & 1.602106 & -1.330867\end{array}$

$\begin{array}{llll}6 & -6.429844 & 0.554880 & 0.296915\end{array}$

$\begin{array}{lllll}6 & -5.539222 & -0.359936 & -1.856047\end{array}$

$\begin{array}{llll}6 & -5.937310 & -1.892218 & 0.112858\end{array}$

$\begin{array}{llll}1 & 3.226951 & 1.962161 & -1.105336\end{array}$ 


$$
\begin{array}{lrrr}
1 & 4.342552 & -1.344304 & 1.429790 \\
1 & -4.367663 & 1.464327 & 1.404209 \\
1 & -3.241243 & -1.924672 & -1.013274 \\
1 & 6.956963 & -0.804717 & -1.300008 \\
1 & 5.302723 & -1.081647 & -1.879864 \\
1 & 5.812408 & -1.720300 & -0.309201 \\
1 & 7.400752 & 0.800214 & 0.627287 \\
1 & 6.264459 & -0.078298 & 1.659895 \\
1 & 6.061091 & 1.662332 & 1.409459 \\
1 & 6.753536 & 1.644928 & -1.603623 \\
1 & 5.422100 & 2.576460 & -0.909139 \\
1 & 5.122023 & 1.455128 & -2.254288 \\
\hline 1 & -7.448503 & 0.359854 & -0.055640 \\
\hline 1 & -6.170350 & 1.578371 & 0.003409 \\
1 & -6.444696 & 0.503525 & 1.391437 \\
\hline 1 & -4.908510 & -1.102471 & -2.355698 \\
1 & -5.216298 & 0.632995 & -2.188156 \\
1 & -6.569719 & -0.515090 & -2.197353 \\
1 & -5.317518 & -2.680285 & -0.327063 \\
1 & -6.970812 & -2.059574 & -0.213042 \\
1 & -5.901027 & -2.005750 & 1.202047
\end{array}
$$


Diphenyl carbene

Symmetry C1

triplet state

Zero-point correction $=0.184336($ Hartree/Particle $)$

Sum of electronic and zero-point Energies $=-\mathbf{5 0 1 . 1 1 9 9 3 0}$

$\mathrm{HF}=-501.3042669$

Atomic Coordinates (Angstroms)

$\begin{array}{llll}\text { Type } & \mathrm{X} & \mathrm{Y} & \mathrm{Z}\end{array}$

$\begin{array}{llll}6 & -0.020 & 0.919 & -0.063 \\ 6 & -1.337 & 0.443 & 0.033 \\ 6 & 1.317 & 0.491 & -0.096 \\ 6 & -2.425 & 1.200 & -0.487 \\ 6 & -1.640 & -0.789 & 0.687 \\ 6 & 2.371 & 1.357 & 0.313 \\ 6 & 1.674 & -0.805 & -0.579 \\ 6 & -3.730 & 0.743 & -0.372 \\ 6 & -2.951 & -1.234 & 0.787 \\ 6 & 3.694 & 0.944 & 0.257 \\ 6 & 3.003 & -1.204 & -0.621 \\ 6 & -4.005 & -0.476 & 0.261 \\ 6 & 4.022 & -0.337 & -0.205 \\ 1 & -0.829 & -1.372 & 1.112 \\ 1 & -2.214 & 2.145 & -0.979 \\ 1 & 0.890 & -1.473 & -0.919 \\ 1 & 2.118 & 2.349 & 0.673 \\ 1 & -4.544 & 1.337 & -0.780 \\ 1 & -3.159 & -2.178 & 1.286 \\ 1 & 4.481 & 1.621 & 0.579\end{array}$




$$
\begin{array}{rrrr}
1 & 3.252 & -2.196 & -0.988 \\
1 & -5.028 & -0.829 & 0.347 \\
1 & 5.060 & -0.655 & -0.245
\end{array}
$$

The complete list of authors for Reference 46.

(46) Frisch, M. J.; Trucks, G. W.; Schlegel, H. B.; Scuseria, G. E.; Robb, M. A.; Cheeseman, J. R.; Zakrzewski, V. G.; Montgomery, J. A.; Stratmann, R. E.; Burant, J. C.; Dapprich, S.; Millam, J. M.; Daniels, A. D.; Kudin, K. N.; Strain, M. C.; Farkas, O.; Tomasi, J.; Barone, V.; Cossi, M.; Cammi, R.; Mennucci, B.; Pomelli, C.; Adamo, C.; Clifford, S.; Ochterski, J.; Petersson, G. A.; Ayala, P. Y.; Cui, Q.; Morokuma, K.; Malick, D. K.; Rabuck, A. D.; Raghavachari, K.; Foresman, J. B.; Cioslowski, J.; Ortiz, J. V.; Baboul, A. G.; Stefanov, B. B.; Liu, G.; Liashenko, A.; Piskorz, P.; Komaromi, I.; Gomperts, R.; Martin, R. L.; Fox, D. J.; Keith, T.; Al-Laham, M. A.; Peng, C. Y.; Nanayakkara, A.; Challacombe, M.; Gill, P. M. W.; Johnson, B.; Chen, W.; Wong, M. W.; Andres, J. L.; Gonzalez, C.; Head-Gordon, M.; Replogle, E. S.; Pople, J. A. GAUSSIAN 98; Gaussian, Inc., Pittsburgh PA, 1998. 